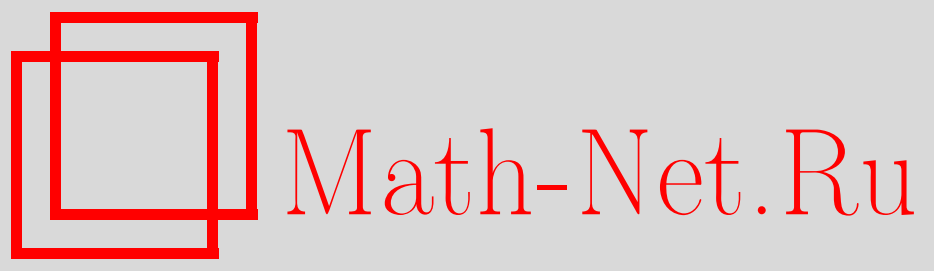

М. Г. Васин, О некоторых деталях описания неупорядоченной конденсированной системы с помощью теории дефектных состояний ориентационного порядка, ТМФ, 2009, том 161, номер 2, 267-277

DOI: https://doi.org/10.4213/tmf6437

Использование Общероссийского математического портала Math-Net.Ru подразумевает, что вы прочитали и согласны с пользовательским соглашением http: //www . mathnet.ru/rus/agreement

Параметры загрузки:

IP : 3.89 .185 .249

26 апреля 2023 г., 16:42:57

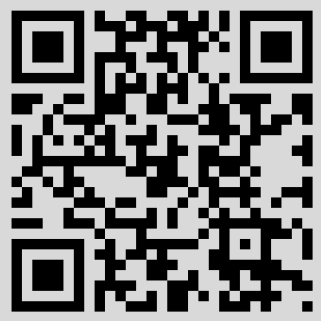




\title{
О НЕКОТОРЫХ ДЕТАЛЯХ ОПИСАНИЯ НЕУПОРЯДОЧЕННОЙ КОНДЕНСИРОВАННОЙ СИСТЕМЫ С ПОМОЩЬЮ ТЕОРИИ ДЕФЕКТНЫХ СОСТОЯНИЙ ОРИЕНТАЦИОННОГО ПОРЯДКА
}

\begin{abstract}
Неупорядоченная конденсированная система описана с помощью теории дефектных состояний ориентационного порядка как упругая среда с линейными топологическими особенностями. Показано, что поля упругих напряжений, создаваемые линейными дисклинациями, являются абелевыми. В квазистационарном линейном приближении получены выражения для тензорных потенциалов линейных дислокаций и дисклинаций. Показано, что $\alpha$-релаксация и $\beta$-релаксация в переохлажденной жидкости могут быть описаны с помощью теории дефектных состояний ориентационного порядка как релаксационные процессы в дисклинационной и дислокационной подсистемах соответственно.
\end{abstract}

Ключевые слова: неупорядоченная структура, топологическая особенность, нарушение $S O(3)$-симметрии.

\section{1. ВВЕДЕНИЕ}

Теория дефектных состояний ориентационного порядка была предложена Нельсоном [1] для описания неупорядоченных конденсированных систем таких, как стекла и жидкости. В ее основе лежит предположение о том, что в большинстве неупорядоченных конденсированных систем существуют наиболее “выгодные" в смысле энергии взаимодействия локальные упаковки атомов (или молекул), определяемые либо длинами и направлениями валентных связей, либо размерами атомных и ионных радиусов и т. п. Ненарушенный локальный порядок в таких системах описывается некоторой группой трансляций и вращений, а неупорядоченность обусловлена нарушением исходной локальной симметрии.

Несмотря на то что теория была предложена более двадцати лет назад, она до сих пор остается на начальной стадии развития. Причиной этого, по-видимому, является довольно сложный математический аппарат теории и доминирование в этой

*Физико-технический институт УрО РАН, Ижевск, Россия. E-mail: dr_vasin@mail.ru 
области физики численных методов. Однако необходимость изучения фундаментальных свойств неупорядоченных конденсированных систем требует развития аналитических методов их теоретического описания. Поэтому интерес к различным теориям жидкого и аморфного состояний вещества сохраняется, в том числе и к теории дефектных состояний ориентационного порядка [2].

В настоящей работе обсуждаются вопросы теоретико-полевого описания дисклинационных и дислокационных полей. Показано, что в теории дефектных состояний ориентационного порядка это описание может быть значительно упрощено благодаря учету нарушения исходной $S O(3)$-симметрии калибровочной теории. В разделе 2 кратко изложены основные положения модели дефектных состояний ориентационного порядка. В разделе 3 даны основные сведения о калибровочной теории дефектов, используемой для описания неупорядоченных конденсированных систем в изучаемой модели. В разделе 4 обсуждаются причины нарушения $S O(3)$-симметрии калибровочной теории дефектов при ее использовании в модели дефектных состояний ориентационного порядка. В разделе 5 выведены выражения для дисклинационного и дислокационного полей и показано, что различие взаимодействий в дислокационной и дисклинационной подсистемах приводит к разной релаксационной динамике.

\section{2. МОДЕЛЬ}

Общие положения теории дефектных состояний ориентационного порядка вещества можно сформулировать следующим образом. При высоких температурах система представляет собой изотропное (квазигазовое) состояние конденсированного вещества, которое характеризуется отсутствием какого-либо ближнего упорядочения атомов и, как следствие, локально $S O(3)$-симметрично. При понижении температуры или увеличении давления в системе происходит формирование ближнего порядка, локально-симметричного относительно преобразований некоторой дискретной группы симметрии. Поскольку эта симметрия определяется только межатомным взаимодействием, естественно предположить, что в большинстве случаев она не относится к федоровским группам, поэтому основное состояние таких локально-упорядоченных систем является некристаллическим (отсутствует дальний трансляционный порядок) и бесконечно вырожденным вследствие присутствия геометрических фрустраций.

Согласно модели дефектных состояний ориентационного порядка основными структурными элементами в неупорядоченном конденсированном веществе являются топологические особенности (дислокации и дисклинации). Образование топологических особенностей обусловлено необходимостью сохранения сплошности структуры при минимальной энергии взаимодействия между элементами этой структуры. Важно отметить, что дисклинации и дислокации - это топологические объекты, и их свойства такие, как, например, зависимость взаимодействия от расстояния, определяются прежде всего топологическими параметрами системы. 
Основным недостатком рассматриваемого метода считается то, что его применимость ограничена малыми концентрациями топологических особенностей. Такое мнение основано, прежде всего, на плохом количественном согласии дислокационных теорий плавления кристаллов с экспериментом. Однако, несмотря на кажущееся сходство, модель дефектных состояний ориентационного порядка принципиально отличается от моделей кристаллического беспорядка. Важно подчеркнуть, что в отличие от дефектов в кристаллах, увеличивающих свободную энергию системы, топологические особенности в рассматриваемой модели являются необходимыми структурными элементами, уплотняющими упаковку и делающими неупорядоченную структуру энергетически более предпочтительной по сравнению с кристаллической [1].

Чтобы разрушить топологически устойчивые дефекты, необходимы значительные энергетические затраты. Однако вследствие фрустрации и сильных тепловых флуктуаций система топологических дефектов подвижна. Это движение представляет собой коллективное движение атомов, благодаря которому неупорядоченное конденсированное вещество обладает текучестью при сохраняющейся сплошности структуры.

\section{3. ЛАГРАНЖКИН КАЛИБРОВОЧНОЙ ТЕОРИИ ДЕФЕКТОВ}

Методом описания неупорядоченных молекулярных систем в модели дефектных состояний ориентационного порядка является калибровочная теория дефектов, базирующаяся на математическом аппарате физики калибровочных полей. Этот подход предполагает фундаментальную роль дефектов в формировании свойств неупорядоченных конденсированных систем. Его основы были заложены в работах [1], $[3]-[5]$.

В наиболее фундаментальной формулировке, предложенной Кадичем и Эделеном [4], континуальная теория дефектов рассмотрена в обобщенной трактовке калибровочных полей Янга-Миллса. Это теория дефектов, непрерывно распределенных в материале. Для того чтобы записать лагранжиан конденсированной системы в этом представлении, рассмотрим стандартную теорию упругой среды. Простейший лагранжиан, описывающий упруго-деформированную систему, имеет вид

$$
L_{0}=\frac{1}{2} \rho_{0} \partial_{t} u_{i} \partial_{t} u_{i}-\frac{1}{8}\left[\lambda\left(u_{i i}\right)^{2}+2 \mu u_{i j} u_{i j}\right]
$$

где $u_{i}(\bar{r}, t)$ - поле упругих деформаций, $\lambda$ и $\mu$ - коэффициенты Ламе (модули упругости и сдвига соответственно), $d u_{i}(\bar{r}, t)$ - относительное смещение близких элементов среды, $\rho_{0}-$ плотность (для простоты предполагается, что эта величина постоянна), $u_{i j}=C_{i j}-\delta_{i j}=\partial_{i} u_{l} \partial_{j} u_{l}-\delta_{i j}-$ тензор относительной деформации. Латинские буквы $a, b, \ldots$ (все, кроме $t$ ) используются в настоящей работе для обозначения пространственных компонент, $\{i\}=\{1,2,3\}, t$ - временна́я компонента, а греческие буквы $\alpha, \beta, \ldots$ используются для обозначения полного набора компонент, включающего время: $\{\alpha\}=\{1,2,3, t\}$. 
В соответствии с калибровочной теорией дефектов пластическую деформацию вещества можно рассматривать как нарушение однородности действия группы симметрии $S O(3) \triangleright T(3)$. Для учета этого нарушения однородности в лагранжиан пластически деформированных систем вводятся компенсирующие поля: тензорный потенциал калибровочного поля дисклинации $A_{a}^{\alpha}$, представляющий собой поворотную часть тензора пластической деформации, и тензорный потенциал калибровочного поля дислокации $\varphi_{b}^{i}$, представляющий собой трансляционную часть тензора пластической деформации. Тогда обычные производные становятся ковариантными:

$$
\partial_{\alpha} u_{i} \rightarrow B_{\alpha i}=\partial_{\alpha} u_{i}+\varepsilon_{i j l} A_{\alpha j} u_{l}+\varphi_{\alpha i},
$$

где $\varepsilon_{i j l}$ - три генерирующие матрицы группы $S O(3)$, а исходный лагранжиан $L_{0}$ заменяется новым лагранжианом

$$
L=L_{0}+s_{\varphi} L_{\varphi}+s_{A} L_{A}
$$

где $s_{\varphi}, s_{A}$ - параметры системы. Первое слагаемое описывает упругие свойства вещества:

$$
L_{0}=\frac{1}{2} \rho_{0} B_{i t} B_{i t}-\frac{1}{8}\left[\lambda\left(E_{i i}\right)^{2}+2 \mu E_{i j} E_{i j}\right],
$$

где $E_{i j}=B_{l i} B_{l j}-\delta_{i j}-$ ковариантный тензор относительной деформации. Второе слагаемое

$$
s_{\varphi} L_{\varphi}=-\frac{1}{2} s_{\varphi} D_{i \alpha \beta} D_{i \alpha \beta}
$$

соответствует вкладу дислокационной подсистемы,

$$
\begin{aligned}
D_{i \alpha \beta} & =\partial_{\alpha} \varphi_{i \beta}-\partial_{\beta} \varphi_{i \alpha}+\varepsilon_{i l j}\left(A_{l \alpha} \varphi_{j \beta}-A_{l \beta} \varphi_{j \alpha}+F_{l \alpha \beta} u_{j}\right) \\
F_{i \alpha \beta} & =\partial_{\alpha} A_{i \beta}-\partial_{\beta} A_{i \alpha}+\varepsilon_{i j l} A_{j \alpha} A_{l \beta} .
\end{aligned}
$$

Третье слагаемое

$$
s_{A} L_{A}=-\frac{1}{2} s_{A} F_{i j l} F_{i j l}+\frac{1}{2 \zeta} s_{A} F_{i t t} F_{i t t}
$$

является вкладом в лагранжиан свободного дисклинационного поля. Поля ЯнгаМиллса $A_{i \alpha}$ и $\varphi_{i \alpha}$ описывают дисклинации и дислокации соответственно. Этот подход позволяет записать полную систему уравнений, представляющую одновременно как эволюцию напряжений в среде, так и динамику дефектов.

\section{4. НАРУШЕНИЕ $S O(3)$-СИММЕТРИИ ЛАГРАНЖКАНА}

Применение описанной выше калибровочной теории дефектов затруднено тем обстоятельством, что в общем случае она является неабелевой [2], [4], т. е. калибровочные поля не коммутируют: $\left[A_{i \alpha} A_{i \beta}\right] \neq 0$. Об этом упоминается во многих работах, посвященных этой теории. Однако внимательное рассмотрение этой проблемы позволяет убедиться, что теория дефектных состояний ориентационного порядка в трехмерном пространстве будет абелевой. 
Дело в том, что $S O(3)$ - это группа симметрии изотропного трехмерного пространства. Само же понятие дефекта подразумевает некоторую локальную анизотропию системы, выражающуюся в существовании параметра порядка $Q$. Группа симметрии параметра порядка $G$ не совпадает с $S O(3)$, поскольку определяется видом потенциальной энергии системы $V(Q)$. Множество значений $M$ параметра порядка (область изменения), минимизирующих потенциал $V(Q)$, называется пространством внутренних состояний, причем $M \sim S O(3) / G$, где $G$ - группа симметрии параметра порядка (группа изотропии) [6]. Все точки множества $M$ эквивалентны в том смысле, что каждая из них может быть получена из другой $S O(3)$-преобразованием. Однако в качестве основного состояния может реализоваться только одна из них. При конденсации система стремится перейти в основное состояние, в результате происходит спонтанное нарушение симметрии $S O(3) \rightarrow G$, и конденсированная система остается инвариантной относительно преобразований $G$. Поскольку локальная симметрия не всегда топологически совместима с какой-либо трансляционной симметрией, иногда она будет нарушаться. Локальные нарушения именно этой группы симметрии, а не $S O(3)$, соответствуют дефектам.

В системах с $S O(3)$-симметричным параметром порядка, например в газе, в качестве параметра порядка которого рассматривается плотность, дефектов просто не существует, поскольку $\pi_{1}(S O(3) / S O(3))=\pi_{2}(S O(3) / S O(3))=1$. Если параметром порядка является спин с группой симметрии $G=S O(2)$, то эта модель представляет собой модель Джорджи-Глэшоу, в которой из-за нарушения симметрии $S O(3) \rightarrow S O(2)$ калибровочные поля становятся абелевыми. Следует отметить, что в этой модели могут существовать только точечные топологические дефекты, в то время как топологически устойчивых линейных дефектов быть не может, поскольку $\pi_{1}(S O(3) / S O(2))=\pi_{1}\left(S_{2}\right)=1$. Топологически устойчивые линейные дефекты возникают в теории, описывающей одноосные нематики, поскольку параметром порядка в ней является директор, обладающий группой симметрии $S O(2) \times \mathbb{Z}_{2}$, и $\pi_{1}\left(S O(3) / S O(2) \times \mathbb{Z}_{2}\right)=\pi_{1}\left(\mathbb{R} P_{2}\right)=\mathbb{Z}_{2}$. Эта теория, так же как и предыдущая, является абелевой. Поэтому ее успешно используют в физике жидких кристаллов, полагая взаимодействие между топологическими дефектами эквивалентным взаимодействию электрических токов.

В конденсированных системах причиной нарушения $S O(3)$-симметрии является возникновение локального ориентационного порядка. Нарушенная симметрия есть симметрия ориентационного упорядочения, которая в общем случае определяется свойствами химических межмолекулярных и межатомных связей. Как было отмечено при формулировке основных положений модели, совершенно не обязательно, чтобы эта симметрия принадлежала к числу кристаллографических групп симметрии. В системах со сферически-симметричным потенциалом взаимодействия наиболее энергетически выгодной локальной упаковкой является тетраэдрическая упаковка, определяющая икосаэдрическую симметрию ближнего порядка. Известно, что группа икосаэдра Y в $S O(3)$ изоморфна конечной группе собственных вращений пространства $\mathbb{R}^{3}$, имеющей три орбиты [7], каждая из которых соответствует 
вращению вокруг одной из трех осей симметрии. Поскольку каждая из орбит совпадает с соответствующим пространством внутренних состояний системы, то все группы симметрии этих внутренних состояний будут дискретными подгруппами группы $S O(2) \times \mathbb{Z}_{2}$. Отсюда следует, что в политетраэдрической системе могут существовать три типа дисклинаций, и все они описываются абелевыми полями. В результате лагранжиан освобождается от нелинейных вкладов $A_{i j}$, характерных для теории Янга-Миллса, т. е. вместо

$$
F_{i \alpha \beta}=\partial_{\alpha} A_{i \beta}-\partial_{\beta} A_{i \alpha}+\varepsilon_{i j l} A_{j \alpha} A_{l \beta}
$$

получаем

$$
F_{i \alpha \beta}=\partial_{\alpha} A_{i \beta}-\partial_{\beta} A_{i \alpha},
$$

что приводит к значительному упрощению анализа рассматриваемых систем.

\section{5. ПОЛЯ ЛИНЕЙНЫХ ТОПОЛОГИЧЕСКИХ ОСОБЕННОСТЕЙ И ИХ МОМЕНТЫ В КВАЗИСТАЦИОНАРНОМ ЛИНЕЙНОМ ПРИБЛИЖЕНИИ}

Для вывода тензорных потенциалов линейных топологических дефектов сделаем несколько приближений. Прежде всего, ограничимся рассмотрением квазистационарного случая $\left(\partial_{t} A_{i \alpha}=0\right)$, поскольку далее нас будут интересовать только медленные процессы релаксации. Кроме того, будем рассматривать только типичные для аморфных систем малоугловые дисклинации с малым индексом Франка $\nu \simeq 0.2$. Масштабный параметр системы равен этой величине, поскольку

$$
\nu=\left|\int_{\Gamma} \varepsilon_{i l k} \nabla_{l} A_{k j} d S_{j}\right| .
$$

Если считать генераторы группы $S O(3) \triangleright T(3)$ не зависящими от масштабного параметра, то он должен войти в компенсирующие поля [4]: $A_{i j} \sim \nu, \varphi_{i} \sim \nu$. Поскольку $\nu$ - малый параметр, рассматриваемая теория может быть линеаризована. Линейное приближение эквивалентно адиабатическому, поскольку, как будет показано ниже, дисклинационное и дислокационное поля становятся независимыми друг от друга. Это приближение является разумным, поскольку времена релаксации дисклинационной подсистемы значительно превышают времена релаксации дислокационной подсистемы.

Для определения тензорного потенциала малого сегмента линейной дисклинации минимизируем вклад от свободных дисклинаций в лагранжиан

$$
L_{A}=\frac{1}{4} F_{\alpha i j} F_{\alpha i j}
$$

В работе [8] было показано, что квазистационарное решение для тензорного потенциала калибровочного поля сегмента линейной дисклинации $d l_{\alpha}$ имеет вид

$$
A_{i k}(\vec{x})=\frac{\nu}{2} \varepsilon_{i k j} \frac{x_{j}}{x^{3}} d l_{i}
$$


где $\vec{x}$ - вектор, определяющий положение точки пространства относительно участка дисклинации. Следует заметить, что в этом выражении нет суммирования по индексу $i$.

В неупорядоченной системе сеть топологических особенностей имеет сложную запутанную структуру. Поэтому задача теоретического описания этой системы, несмотря на сделанные выше приближения, остается сложной. Однако ее можно значительно упростить, учитывая высокие плотность и подвижность топологических особенностей. Два этих свойства топологических “дефектов" приводят к их взаимной экранировке, уменьшающей упругую энергию системы и минимизирующей плотность топологического заряда. С учетом экранировки тензорный потенциал поля дисклинационной подсистемы определяется топологическим моментом $Q_{i j}(r)$ (см. ниже приложение):

$$
A_{i a}(\vec{x})=\frac{Q_{i a}^{\mathrm{R}}}{x^{3}}
$$

Учитывая выражения (1), можно получить поле линейной дислокации следующим образом. В линейном приближении можно пренебречь первыми двумя членами в скобках, так как произведение $A \varphi \sim \varepsilon^{2}$. Тогда

$$
D_{i \alpha \beta}=\partial_{\alpha} \varphi_{i \beta}-\partial_{\beta} \varphi_{i \alpha}+\varepsilon_{i l j} F_{l \alpha \beta} u_{j}
$$

где

$$
F_{i \alpha \beta}=\partial_{\alpha} A_{i \beta}-\partial_{\beta} A_{i \alpha}
$$

Используя соотношение (4), получаем

$$
D_{i \alpha \beta}=\partial_{\alpha} \varphi_{i \beta}-\partial_{\beta} \varphi_{i \alpha}+\int \varepsilon_{i l j}\left(\partial_{\alpha} A_{l \beta}(\bar{x}-\bar{r})-\partial_{\beta} A_{l \alpha}(\bar{x}-\bar{r})\right) x_{j} d \vec{r}=\partial_{\alpha} \varphi_{i \beta}-\partial_{\beta} \varphi_{i \alpha}
$$

Отсюда следует, что в линейном приближении дислокационное и дисклинационное поля независимы друг от друга. Это приближение эквивалентно пренебрежению процессами рождения и уничтожения дислокаций на дисклинациях, а поля линейных дислокаций имеют тот же вид, что и дисклинационные поля:

$$
\varphi_{i j}(\vec{x})=\varepsilon_{i j k} \frac{x_{k}}{x^{3}} d l_{i}
$$

В результате можно получить тензорные потенциалы дислокационных и дисклинационных полей произвольной конфигурации.

\section{6. ВЗАИМОДЕЙСТВИЕ МЕЖДУ ТОПОЛОГИЧЕСКИМИ МОМЕНТАМИ}

Несмотря на то что тензорные потенциалы линейных дислокаций и дисклинаций имеют одинаковый вид, в упругое взаимодействие они входят по-разному. Как будет показано ниже, это обстоятельство определяет существенное различие в релаксационной динамике дислокационной и дисклинационной подсистем.

5 Теоретическая и математическая физика, т. 161, № 2, 2009 г. 
Поле напряжений создается дисклинациями и дислокациями. Ограничиваясь первым порядком малости и оставляя только недиагональные элементы, запишем тензор упругой деформации:

$$
\sigma_{a i} \simeq \mu E_{a i}=\mu\left(B_{a j} B_{j i}-\delta_{a i}\right)=\mu\left[\varepsilon_{\alpha a l} x_{l} A_{\alpha i}+\varepsilon_{\alpha i l} x_{l} A_{\alpha a}+\varphi_{a i}+\varphi_{i a}+\partial_{i} u_{a}+\partial_{a} u_{i}\right] .
$$

Энергия упругого взаимодействия

$$
U^{\mathrm{E}}=\frac{1}{2} \int E_{a i} \sigma_{a i} d V=U^{\mathrm{R}}+U^{\mathrm{T}}+U^{\mathrm{RT}}
$$

содержит вклады от междисклинационных $\left(U^{\mathrm{R}}\right)$ и междислокационных $\left(U^{\mathrm{T}}\right)$ взаимодействий, а также от взаимодействий дислокаций с дисклинациями $\left(U^{\mathrm{RT}}\right)$. Несмотря на то что при количественных расчетах третьим вкладом нельзя пренебрегать, для качественного анализа системы достаточно рассмотреть только первые два из них.

Как отмечалось выше, экранировка приводит к минимизации локальных топологических зарядов в системе. Если разделить весь объем системы на ячейки таким образом, чтобы их топологические заряды были минимальными $(q(\vec{r}) \rightarrow 0)$, то энергию взаимодействия сети линейных топологических особенностей можно представить как энергию системы локальных топологических моментов этих ячеек:

$$
U^{\mathrm{R}}=\frac{\mu}{2} \varepsilon_{\alpha a l} x_{l} A_{i}^{\alpha} \varepsilon_{\gamma a j} x_{j}^{\prime} A_{i}^{\prime \gamma}=\frac{a^{2}}{2} \sum_{\vec{r}, \vec{r}^{\prime}} Q_{i l}^{\mathrm{R}}(\vec{r}) J_{i j l k}^{\mathrm{R}}\left(\vec{r}-\vec{r}^{\prime}\right) Q_{j k}^{\mathrm{R}}\left(\vec{r}^{\prime}\right),
$$

где векторы $\vec{r}$ и $\vec{r}^{\prime}$ задают положения моментов, а тензор взаимодействия между ними имеет вид

$$
J_{l k i j}^{\mathrm{R}}(\vec{r}) \approx \mu \frac{\delta_{i j}}{2|\vec{r}|^{3}}\left(\delta^{l k}-3 \frac{r^{l} r^{k}}{r^{2}}\right) .
$$

Аналогично энергия упругого взаимодействия системы дислокационных топологических моментов может быть представлена как

$$
U^{\mathrm{T}}=\frac{\mu}{2} \int \varphi_{a i} \varphi_{a i}^{\prime} d V=\frac{a^{2}}{2} \sum_{\vec{r}, \vec{r}^{\prime}} Q_{i l}^{\mathrm{T}}(\vec{r}) J_{i j l k}^{\mathrm{T}}\left(\vec{r}-\vec{r}^{\prime}\right) Q_{j k}^{\mathrm{T}}\left(\vec{r}^{\prime}\right),
$$

где

$$
J_{l k i j}^{\mathrm{T}}(\vec{r}) \approx \mu \frac{\delta_{i j}}{2|\vec{r}|^{5}}\left(\delta^{l k}-3 \frac{r^{l} r^{k}}{r^{2}}\right) .
$$

Сравнивая выражения для междисклинационного и междислокационного взаимодействий, видим, что первое является дальнодействующим взаимодействием, $\mathbf{J}^{\mathrm{R}}(r) \sim 1 / r^{3}$, а второе - короткодействующим, $\mathbf{J}^{T}(r) \sim 1 / r^{5}, 5>d=3$. Это очень важное различие, поскольку оно является причиной различия релаксационной динамики дисклинационной и дислокационной подсистем.

Чтобы это показать, представим функционал свободной энергии системы дефектов в виде функционала свободной энергии теории фрустрационно-ограниченных доменов [9]:

$$
U^{\mathrm{R}}=\int\left\{\frac{1}{2}\left|\partial_{\mu} \mathbf{Q}^{\mathrm{R}}(\vec{x})\right|^{2}+\frac{1}{2} \tau\left|\mathbf{Q}^{\mathrm{R}}(\vec{x})\right|^{2}\right\} d^{3} x+\frac{1}{2} \iint \mathbf{Q}^{\mathrm{R}}(\vec{x}) \mathbf{J}^{\mathrm{R}}(|\vec{x}-\vec{y}|) \mathbf{Q}^{\mathrm{R}}(\vec{y}) d^{3} x d^{3} y,
$$


где $\mathbf{Q}$ - локальный топологический момент (параметр порядка). Первое слагаемое - близкодействующее взаимодействие, обусловленное непрерывностью поля $\mathbf{Q}$, второе слагаемое - слабое дальнодействующее взаимодействие, которое описывает взаимодействие между дисклинационными моментами. Хорошо известно, что такие дальнодействующие взаимодействия, как кулоновское, или диполь-дипольное, приводят к возникновению в системе конкурирующих состояний и к ее фрустрированию [9], [10]. Таким образом, дисклинационная подсистема вследствие дальнодействующего взаимодействия также будет геометрически фрустрированной. Существует большое количество работ, в которых показано, что релаксационная динамика в таких системах является медленной [2], [11], а температурная зависимость времени релаксации описывается законом Фогеля-Фульчера-Таммана, $\tau \sim$ $e^{\mathrm{const} /\left(T-T_{0}\right)}$. Таким образом, по определению релаксация дисклинационной подсистемы представляет собой $\alpha$-релаксацию.

С другой стороны, короткодействующие взаимодействия в дислокационной подсистеме не могут привести к ее фрустрированию, поэтому динамика релаксации этой подсистемы имеет обычный аррениусовский характер, $\tau \sim e^{\text {const } / T}$, и является $\beta$-релаксацией. Такое различие в динамике подсистем хорошо согласуется с современными представлениями о переохлажденном жидком состоянии вещества [12]. Степени свободы, связанные с движением дисклинаций, по своей природе являются ротационными и соответствуют коллективному движению атомов, в то время как дислокационные степени свободы являются трансляционными. Эти различия приводят к разделению релаксации переохлажденной жидкости на два типа: первый связан с ротационным ( $\alpha$-релаксация), а второй - с трансляционным ( $\beta$-релаксация) типами диффузии соответственно.

\section{7. ЗАКЛЮЧЕНИЕ}

Важная особенность рассмотренного выше подхода заключается в возможности последовательного описания плотных неупорядоченных конденсированных веществ таких, как стекла и переохлажденные жидкости, связывающего макроскопические свойства вещества с симметрийными свойствами его структуры на уровне ближнего порядка. Основным положением настоящей работы является утверждение о том, что наличие нетривиальной симметрии локального порядка в реальной молекулярной системе приводит к нарушению $S O(3)$-симметрии лагранжиана идеальной калибровочной теории дефектов и значительно упрощает аналитическое описание. В приближении первого порядка малости дисклинационное и дислокационное поля независимы друг от друга. Это также упрощает анализ, но в то же время позволяет сохранить важные свойства системы. Одним из таких свойств является разделение релаксации на ротационную $\alpha$-релаксацию и трансляционную $\beta$-релаксацию.

Теория дефектных состояний ориентационного порядка представляется весьма полезной для описания твердых аморфиков и других неупорядоченных конденсированных систем. Основной недостаток этой теории, не позволяющий в полной мере 
использовать ее для описания жидкого состояния - условие малой концентрации топологических особенностей. Кроме того, даже при низких температурах в системе возможно образование метастабильных неустойчивых дефектов, количество которых возрастает при увеличении температуры. Тем не менее можно ожидать, что рассмотренная теория применима для описания жидкости в ограниченной области сильного переохлаждения и может быть использована при теоретическом описании перехода жидкость-стекло.

\section{ПРИЛОЖЕНИЕ}

Для того чтобы определить топологический момент дисклинационного поля, рассмотрим тензорный потенциал произвольной системы замкнутых линейных дисклинаций, заключенных в произвольном объеме $V$ :

$$
A_{i a}=e_{i} \frac{\nu}{2} \varepsilon_{i a k} \int \frac{x^{k}}{x^{3}} l_{i} d V,
$$

где $x$ - расстояние от элемента дисклинации $l_{i} d V$ до точки наблюдения, $e_{i}-$ единичный вектор. Если выбрать внутри рассматриваемой системы дисклинаций произвольную точку - центр "токов" - и обозначить через $\vec{R}$ вектор, проведенный от этой точки до точки наблюдения, а через $\vec{r}$ - вектор, проведенный от этой точки до элемента дисклинации $(\vec{R}=\vec{x}+\vec{r})$, то при условии $r \ll x$ в разложении выражения (5) можно ограничиться двумя первыми членами:

$$
\begin{aligned}
A_{i a}= & e_{i} \frac{\nu}{2} \varepsilon_{i a k} \frac{R_{k}}{R^{3}} \int l_{i} d V+e_{i} \frac{\nu}{2} \varepsilon_{i a k} \int \frac{r_{k}}{R^{3}} l_{i} d V+ \\
& +e_{i} \frac{3 \nu}{2} \varepsilon_{i a k} \int \frac{R_{k} R_{s} r_{s}}{R^{5}} l_{i} d V+\cdots
\end{aligned}
$$

Отношение двух последних слагаемых к первому по порядку величины равно $l / x$, где $l$ - поперечный размер объема $V$. Поэтому на расстояниях $x>l$, на которых справедлива эта формула, последние два слагаемых малы по сравнению с первым, и приближенно можно считать, что

$$
A_{i a}=e_{i} \frac{\nu}{2} \varepsilon_{i a k} \frac{R_{k}}{R^{3}} \int l_{i} d V
$$

Однако в случае системы экранирующих друг друга замкнутых топологических дефектов, заключенных в объеме $V$, т. е. когда суммарный топологический заряд дисклинаций, проходящих через ограничивающую этот объем поверхность $S$, равен нулю,

$$
\nu \int l_{i} d V=0
$$

первое и третье слагаемые выражения (6) исчезают. В результате имеем

$$
A_{i a}=\pi a^{2} \nu\left(\delta_{i a}-3 e_{i} e_{a}\right) \frac{1}{R^{3}}+\cdots
$$


Таким образом, основным параметром, характеризующим систему замкнутых линейных дисклинаций, является топологический момент $Q_{a}^{i}=\pi a^{2} \nu\left(\delta_{a}^{i}-3 e^{i} e_{a}\right)$, а тензорный потенциал этой системы на больших расстояниях равен

$$
A_{i a}=\frac{Q_{i a}^{\mathrm{R}}}{R^{3}}
$$

где $R$ - расстояние от точки наблюдения до системы токов, характеризуемой ее топологическим моментом $Q^{\mathrm{R}}$. Аналогично можно выразить тензорный потенциал дислокационного поля через дислокационный топологический момент $Q^{\mathrm{T}}$.

Благодарности. Настоящая работа выполнена при поддержке РФФИ (гранты № 07-02-00110-а и № 07-02-96045-р_урал_а).

\section{Список литературы}

[1] D. R. Nelson, Phys. Rev. B, 28:10 (1983), 5515-5535.

[2] G. Tarjus, S. A. Kivelson, Z. Nussinov, P. Viot, J. Phys. C, 17:50 (2005), R1143-R1182.

[3] В. А. Лихачев, А. Е. Волков, В. Е. Шудегов, Континуалъная теория дефектов, ЛГУ, Л., 1986.

[4] А. Кадич, Д. Эделен, Калибровочная теория дислокачий и дисклинаций, Мир, М., 1987.

[5] N. Rivier, D. M. Duffy, "Line defects and the glass transition", Numerical Methods in the Study of Critical Phenomena, Springer Ser. Synergetics, 9, eds. J. Della Dora, J. Demongeot, B. Lacolle, Springer, Berlin, 1981, 132-142.

[6] М. И. Монастырский, Топология калибровочных полей и конденсированных сред, ПАИМС, М., 1995.

[7] С. П. Новиков, И. А. Тайманов, Современные геометрические структуры и поля, МЦHMO, M., 2005.

[8] M. G. Vasin, V. I. Lady'anov, J. Phys. C, 17:14 (2005), S1287-S1292.

[9] D. Kivelson, G. Tarjus, J. Chem. Phys., 109:13 (1998), 5481-5486.

[10] P. A. Prudkovskii, A. N. Rubtsov, M. I. Katsnelson, Europhys. Lett., 73:1 (2006), 104-109.

[11] M. G. Vasin, Phys. Rev. B, 74:21 (2006), 214116.

[12] P. G. Debenedetti, F. H. Stillinger, Nature, 410:6825 (2001), 259-267.

Поступила в редакцию 2.03.2009 